\title{
Novel Nanostructures of Tin Oxide Synthesized by Spray Pyrolysis
}

\author{
Edgar Guerra-Aguirre, Mario Miki-Yoshida and Francisco Paraguay-Delgado* \\ Departamento de Física de Materiales, Centro de Investigación en Materiales Avanzados S. C. \\ Miguel de Cervantes 120 C. P. 31109. Complejo Industrial Chihuahua, Chih. México. \\ *Corresponding autor: francisco.paraguay@cimav.edu.mx
}

Nowadays the possible applications of nanoestructures materials are extending to the different areas such as the medicine, electronics, sensors, etc. In the particular case of tin oxide, it can have almost immediate applications in toxic gas sensing, such as $\mathrm{CO}$, propane, NOx, with high sensitivity ${ }^{1}$. That is to say, it can have high sensitivity to detect polluting agents in the atmosphere around parts per billion. In this work, we presented the process of the synthesis and the microstructural characterization of the different shapes with nanometrics dimensions, such as particles, rods, strips, spheres and flowers.

The synthesis of these particles has been obtained by the Spray-Pyrolysis ${ }^{2}$ technique; it involves the atomization of a precursor solution (alcoholic solution of tin tetrachloride) into very small discrete droplets. These droplets are subsequently transported through the tube. The tubing was heated at around $900^{\circ} \mathrm{C}$ in a horizontal Lindberg Scientific furnace where the solvent evaporates and the dissolved species react to form the product. The nanostrured materials were obtained on the tubing surface, mostly in a radial direction, i.e. perpendicular to the glass. The advantage of this process is the obtaining of the nanostructure materials to smaller temperature in comparison of the used techniques up to date ${ }^{3}$. In this work a systematic study becomes of the diverse zones of the tube in where we deposited the material, these was retired from the surface for their characterization by the electron microscopy techniques in their modalities of SEM, TEM, STEM using the techniques of SAED, CBED and $\mu \mu \mathrm{D}$. The elementary composition was study by EDS and EELS.

In the figure 1a, we see the structure like-nanoflower of tin oxide obtained by SEM, where the leaves have hollows and have a pyramid-like geometry whose ends are located in the origin, the growth is more or less homogenous. The Figure $1 \mathrm{~b}$ show the homogeneity of the materials synthesized in rods shapes, whose diameter average is around $80 \mathrm{~nm}$ and length between 5 and 15 microns. In these structures we observed some changes in the direction of growth in around 120 degrees.

In figure 2 we see bright field TEM micrographs of a ribbon like in form of $\mathrm{V}$, attached to the same image we can appreciated the corresponding SAED, which demonstrates that the material is monocrystal. Figure $2 b$ shows bright field TEM micrographs of nanoparticles. In fig 3 we can see STEM mode image obtained, where we observed the morphologies of rods, strips and particles with nanometric dimensions. In the figure $3 b$ we appreciated the micrograph in dark field in an STEM mode for high magnification from the zone of ribbons, we can see the changes in the growth direction.

The Spray pyrolysis technique is adequate for the obtaining of nanometric materials of tin oxide, also can be used this method for the synthesis of other oxide materials. By means of the morphologic and microstructural characterization we concluded that, each nanometric form has preferential growth in some zones of obtaining, which mainly depend on the temperature and the thermodynamic process that undergoes the pulverized drops.

\section{Acknowledgements}

We are very grateful to Wilber Antunez, Enrique Torres and Carlos Ornelas for experimental assistance. 

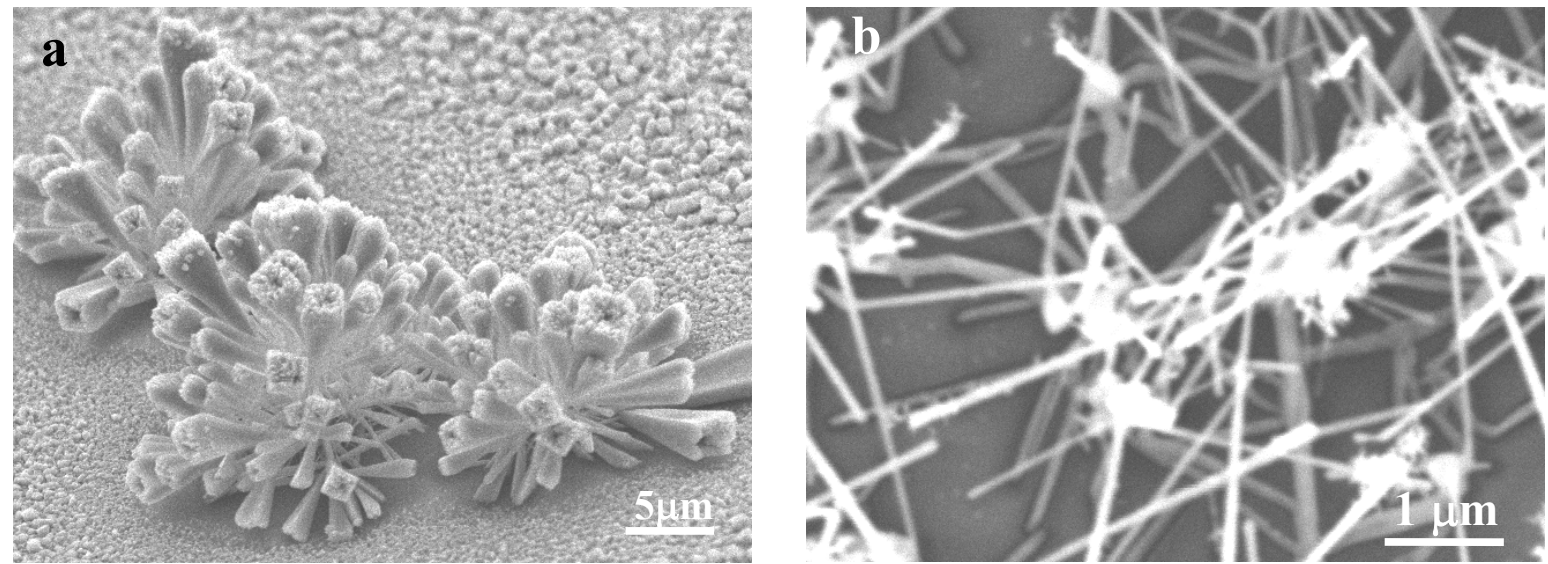

Fig. 1 Secondary electron SEM micrograph of a)nano-flowers and b) nanorods of tin oxide.
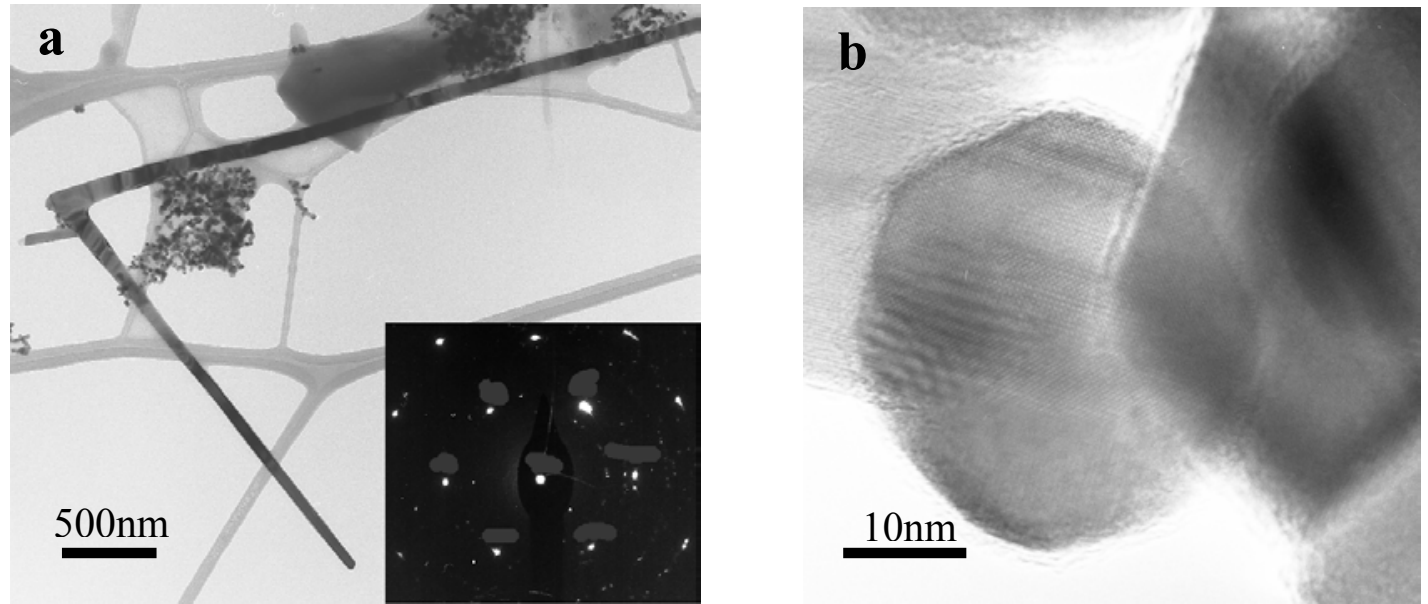

Fig. 2 bright field TEM micrograph of a) nanorod and b) nanoparticle
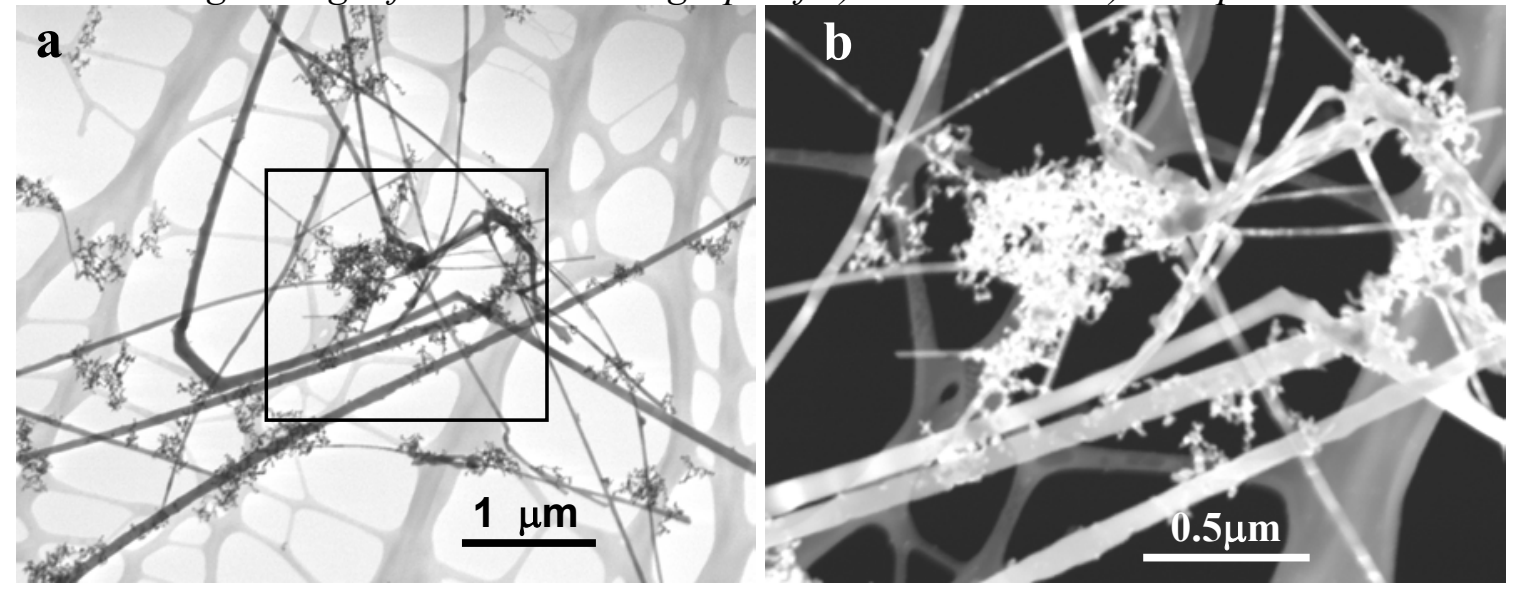

Fig. 3 STEM images of nanoparticles, nanorods and nanoribbons a) bright field and b)

References dark field images respectively.

1 Yuliang Wang, Xuchuan Jiang, and Younan Xia J. Am. Chem. Soc. 2003, 125, 16176-16177

2 F. Paraguay D, W. Estrada L., et. al. Thin Solid Films 350 (1999) 192-202.

3 Y. Chen et al. / Chemical Physics Letters 369 (2003) 16-20. 